\title{
Primary care physicians' utilization and perceptions of genetics services
}

Susan J. Hayflick, MD, M. Patrice Eiff, MD, Leslie Carpenter, MS, MPH, and Judy Steinberger, $P h D$

Purpose: To document primary care physicians' utilization and perceptions of genetics services. Methods: A randomized survey of physicians in the Pacific Northwest. Results: The greatest factor prompting a genetics referral was the patient's interest in the evaluation, and the most common reason not to obtain a consultation was the perception that it was of no benefit to the patient. Genetics consultation was rarely sought for a family history of cancer or for deafness, polycystic kidney disease, or congenital heart disease. Even when uncertain about relative risk, physicians usually counseled a patient themselves rather than referring to a specialist. Conclusion: Primary care physicians need more education about the genetic component of many diseases to provide directly and to refer appropriately for genetics services.

Koy words: Genetics services, primary care, attitudes, perceptions, utilization

Many physicians believe that genetic disease is rare. Although most individual disorders are uncommon, there are thousands of human genetic diseases. ${ }^{1}$ In aggregate, genetic disease is common and accounts for substantial morbidity and mortality..$^{1,2}$ As genetic factors in common diseases are identified, genetics will become even more relevant to the health of an increasing number of individuals. These advances demand commensurate growth in the awareness and recognition of genetic components of disease by physicians and in their preparedness to provide genetics services. This is especially true in today's climate of managed health care, in which primary care physicians play a pivotal role in coordinating services.

Potential barriers to the effective utilization of genetic testing and services are deficiencies in the knowledge of genetics, limited awareness of genetics services, financial pressures within managed care plans, and perceptions of a limited benefit or even risk of harm from these services. Deficiencies in primary care physicians' knowledge of genetics and genetics tests are well documented ${ }^{3-6}$ and have been linked with referral practices. ${ }^{4,7-10}$ These deficiencies are not unexpected because most health care providers were educated and trained before the genetics revolution of the past decade.

In this study, we sought to determine primary care physicians' knowledge of local and regional genetics services, their patterns of use of these services, and their perceptions of the benefits and detriments of these services. To evaluate the direct delivery of genetics services by primary care physicians, we asked about routine genetic screening practices and management of patients with a specific genetic disorder or family history.

\section{METHODS}

The Pacific Northwest region includes Alaska, Idaho, Oregon, and Washington and encompasses a population of nearly 10 million people spread across more than 835,000 square miles. It includes several large metropolitan areas, a substantial rural population, and areas of extreme geographic isolation.
Medical genetics services currently are delivered in academic medical centers, outreach clinics in both metropolitan and rural sites, and offices of private physicians. In Oregon and Washington, statewide services are provided by comprehensive programs within each state. Genetic counselors practice in both Alaska and Idaho, which currently have no resident medical geneticists. Alaska contracts with the University of Washington for clinical genetics services, and Idaho contracts with providers from several western states.

A three-page survey was mailed to all members of the American Academy of Pediatrics, the American College of Obstetrics and Gynecology, and the American College of Physicians who practice in Alaska, Idaho, Oregon, and Washington and to a random sample of approximately half of the physician members of the American Academy of Family Practice. To increase the response rate, a second mailing to nonresponding physicians was sent six weeks after the initial mailing. After an additional eight weeks, each data set was closed. For reasons related to the funding of this study, the family physician survey was completed in January 1995, and the internist, pediatrician, and obstetrician/gynecologist studies were completed in March 1996. New questions were added to the internist, pediatrician, and obstetrician/gynecologist surveys in response to some of the family physician data.

Four surveys, one for each specialty, were developed by genetics and primary care professionals. Although each was tailored to the scope of one specialty, all instruments contained a subset of identical items. Questions addressed perceived availability, benefits, and detriments of genetics services, and current patterns of genetics referral. Direct provision of genetics services by primary care providers was assessed with questions about diagnostic, treatment, and risk assessment practices related to common genetic disorders. Personal and practice demographic items composed the final section of the surveys. All questions were multiple choice with the exception of the open-ended query, "What do
From the Departinents of Molecular and Medical Genetics, Pediatrics, and Family Medicine, Oregon Health Sciences University, Portland, Oregon; and the Department of Obstetrics and Gynecology, University of Washington, Seattle, Washington

Address correspondence to: Susan J. Hayflick, MD, Department of Molecular and Medical Genetics, Oregon Health Sciences University, mail code L103, 3181 SW Sain Jackson Park Road, Portland, Oregon 97201-3098. E-mail: hayflick@ohsu.edu

(c) 1998 Genetics in Medicine. All rights reserved. 1098-3600\$0.00 
you perceive to be the benefits/detriments of a genetics consultation?" All survey instruments can be viewed via the World Wide Web at URL: http://www.ohsu.edu/som-MedGen/pcpsurvey.htm. The internal medicine survey instrument is shown in Appendix 1.

Demographic data collected included gender, year of graduation from medical school, completion of a primary care residency, state in which practicing, community size, practice or panel size, distance to nearest genetics consultant, enrollment in a genetics course in medical school, and status of having taken a continuing medical education course specifically covering genetics.

A database was created (Statistical Package for the Social Sciences, version 6.1.4) for each data set. Responses to each survey item were tabulated and the relationship of selected items was examined by cross-tabulation and tested for significance with the $\chi^{2}$ test. Survey data from each specialty were analyzed separately. Common items from all surveys were compared by physician group.

\section{RESULTS}

Surveys were delivered to 4824 physicians, including 1336 internists, 1227 obstetrician/gynecologists, 1078 pediatricians, and 1183 family physicians. Completed surveys were received from 1642 (34\%) respondents, including 401 internists, 394 obstetrician/gynecologists, 436 pediatricians, and 411 family physicians (Table 1). Based on data from each academy, demographics of respondents were representative of primary care physicians in the Northwest with respect to gender, practice setting, completion of a residency, and, for family physicians, inclusion of obstetrics in their practice. In the Pacific Northwest, family physicians are much more likely to provide obstetrical care than their colleagues from other US regions ( $45 \%$ vs. $26 \%$, American Academy of Family Practice membership data 1995).

Respondents were queried about their utilization of genetics services (Table 2). One in four internists did not know if genetics consultation was available to them. Fifteen percent of internists surveyed knew of no available services and reported no need for additional genetics services.

In the Pacific Northwest, nearly two-thirds of

Table 1

Demographics of respondents ${ }^{\mathrm{a}}$

\begin{tabular}{lcccc}
\hline & FP & IM & OB/GYN & PED \\
\hline Response rate & 35 & 30 & 32 & 40 \\
Residency trained & 81 & 95 & 80 & 94 \\
$\begin{array}{l}\text { Community size: } \\
\quad\end{array}$ & 52 & 34 & 33 & 29 \\
$\quad 50 \mathrm{~K}-100 \mathrm{~K}$ & 12 & 13 & 14 & 16 \\
$>100 \mathrm{~K}$ & 36 & 53 & 53 & 55 \\
Genetics course: & & & & \\
$\quad$ Medical school & 54 & 57 & 55 & 63 \\
CME & 16 & 10 & 37 & 35 \\
\hline${ }^{2} \mathrm{n}=1642$; values are percentages. & &
\end{tabular}

FP, family practice; IM, internal medicine; $O B / G Y N$, obstetrics/gynecology; PED, pediatrics. physicians surveyed reported that they practice within one hour of a genetics consultant (medical geneticist or genetic counselor), and $93 \%$ are within a threehour drive. Despite the large geographic region covered by this study, distance was a barrier to referral for only $15 \%$ of respondents.

The important factors in the decision to refer for genetics consultation and the perceived benefits and detriments are listed in Table 3. The most important factor prompting a genetics referral was the patient's or family's interest in the referral. Malpractice concerns were more important to obstetrician/gynecologists than to other specialists. The leading reason not to obtain genetics consultation was that practitioners "don't see much benefit for the patient." Internists and pediatricians were significantly more

Table 2

Utilization of genetics services $^{a}$

\begin{tabular}{lcccc}
\hline & FP & IM & OB/GYN & PED \\
\hline $\begin{array}{l}\text { Genetic counseling or } \\
\text { consultation available }\end{array}$ & 89 & 59 & 90 & 95 \\
$\begin{array}{l}\text { Additional genetics } \\
\text { services needs }\end{array}$ & 7 & 8 & 4 & 10 \\
$\begin{array}{l}\text { Obtained } \geq 1 \text { genetics } \\
\text { consultation in the } \\
\text { past year }\end{array}$ & $31^{b} / 82^{c}$ & 25 & 95 & 91 \\
\hline
\end{tabular}

"Values are percentages.

${ }^{b}$ Family physicians not practicing obstetrics.

' Family physicians practicing obstetrics.

FP, family practice; IM, internal medicine; OB/GYN, obstetrics/gynecology; PED, pediatrics.

\section{Table 3}

Referral factors and perceptions of genetics consultation $^{a}$

\begin{tabular}{|c|c|c|c|c|}
\hline & FP & IM & $\overline{\mathrm{OB} / G Y N}$ & PED \\
\hline \multicolumn{5}{|c|}{ Factors in decision to refer to genetics: } \\
\hline Family's interest & NQ & 73 & 90 & 94 \\
\hline Severity of the disorder & NQ & 69 & 88 & 89 \\
\hline Malpractice concerns & NQ & 35 & 80 & 42 \\
\hline \multicolumn{5}{|c|}{ Factors in decision not to refer to genetics: } \\
\hline \multicolumn{5}{|l|}{ Don't see much benefit } \\
\hline for patient & 38 & 64 & 48 & 62 \\
\hline \multicolumn{5}{|l|}{ Able to get info } \\
\hline by phone & 35 & 42 & 62 & 63 \\
\hline \multicolumn{5}{|l|}{ Managed care } \\
\hline & & & & \\
\hline \multicolumn{5}{|l|}{ Benefits: } \\
\hline Education/knowledge & 47 & 34 & 50 & 49 \\
\hline Reproductive planning & 28 & 21 & 12 & 33 \\
\hline Better direct care & 3 & 13 & 12 & 22 \\
\hline None & 4 & 10 & 9 & 9 \\
\hline \multicolumn{5}{|l|}{ Detriments: } \\
\hline None & 51 & 38 & 52 & 60 \\
\hline Cost & 15 & 15 & 20 & 16 \\
\hline \multicolumn{5}{|l|}{ Cause undue } \\
\hline worry/anxiety & 18 & 13 & 15 & 7 \\
\hline No practical outcome & 1 & 6 & 4 & 5 \\
\hline
\end{tabular}

percentages.

$\mathrm{FP}$, family practice; IM, internal medicine; OB/GYN, obstetrics/gynecology; $P E D$, pediatrics; NQ, not queried. 
likely than obstetrician/gynecologists or family physicians to select this statement $(P<0.001)$. The second most common reason was that respondents were "able to get information from a consultant by telephone." Managed care plan limits were a barrier to referral for $25 \%$ of internists and pediatricians but only $15 \%$ of obstetrician/gynecologists.

The leading benefits of a genetics consultation reported by all practitioners were education and reproductive planning. Nearly $10 \%$ of respondents specifically wrote "none" when asked what they perceive to be the benefits of a genetics consultation. Among all specialties, issues of high cost and creating undue worry or anxiety were listed as leading detriments of a genetics consultation, and $49 \%$ said there are no detriments of a genetics consultation.

Although genetics consultation was often sought for individuals with dysmorphic features or a family history of a "genetic condition," it was rarely sought for patients with deafness, polycystic kidney disease, congenital heart disease, or a family history of cancer. Pediatricians often did not refer for isolated mental or growth retardation or fetal exposure to alcohol or drugs. Twenty percent of internists reported that they would rarely refer an individual with a family history of Huntington's disease for genetics consultation.

Obstetrician/gynecologists usually referred patients for genetic counseling before fetal chromosome testing, after an abnormal fetal chromosome test, for a family history of a specific genetic disorder, and for patients with features of a genetic condition. In comparison, obstetrician/gynecologists were less likely to refer for a family history of a birth defect or mental retardation. They were also less likely to refer patients whose ethnic background placed them at increased risk for a specific genetic disorder (e.g., Ashkenazi Jewish, Asian), with a majority reporting instead that they routinely discuss carrier screening with these patients. The most common types of patients referred by family physicians were prenatal patients, those with a family history of a birth defect or genetic disorder, and neonates with a birth defect or genetic disorder.

Several questions addressed screening and risk assessment practices by primary care physicians. More than $95 \%$ of physicians reported taking a family history as part of their routine care. Physicians who provided obstetrical care were more likely, than those who did not, to ask about a couple's ethnic background ( $70 \%$ vs. $48 \%, P<0.001)$. Obstetrician/gynecologists and family physicians who inquired about ethnicity were significantly more likely ( $87 \%$ vs. $56 \%, P<0.001$ ) to offer carrier screening for common genetic diseases (Tay-Sachs disease, sickle cell anemia, or alpha thalassemia to patients of Ashkenazi Jewish, African, or Asian descent, respectively) than their colleagues who did not ask. However, family physicians practicing obstetrics, who were just as likely as obstetrician/gynecologists to ask about a couple's ethnic background, were less likely to offer carrier screening. Of physicians providing prenatal care, $94 \%$ routinely offer fetal karyotyping to women older than 34 years of age, and $93 \%$ routinely discussed maternal serum alpha-fetoprotein or triple screen testing with pregnant patients. For patients with a history of prior offspring with a neural tube defect, $91 \%$ of obstetrician/gynecologists discussed preconceptional folate supplements, compared with $78 \%$ if there was no history $(P<0.025)$.

Pediatricians and family practitioners were asked about their routine care of a newborn with obvious features of Down syndrome. Most pediatricians and family physicians ( $89 \%$ and $72 \%$, respectively) reported that they would obtain a karyotype even when they were confident about the clinical diagnosis of Down syndrome. Seventy-one percent of pediatricians and $52 \%$ of family physicians would tell parents the diagnosis before knowing the chromosome results. In the setting of a normal clinical cardiac examination, $65 \%$ of pediatricians and $51 \%$ of family physicians would obtain an echocardiogram on an infant with Down syndrome.

Internists, family physicians, and pediatricians were asked about their approach to evaluating a male with isolated mental retardation. Only $35 \%$ of internists would obtain a two- or three-generation family history, and $13 \%$ would either refer to a genetics clinic or obtain a karyotype. In contrast, $77 \%$ of pediatricians would obtain a family history, and $65 \%$ would either refer to a genetics clinic or obtain a karyotype. Five percent of internists and $11 \%$ of family practitioners would test a male with isolated mental retardation for fragile $\mathrm{X}$ syndrome, compared with $67 \%$ of pediatricians. Eleven percent of family physicians reported being unfamiliar with fragile X syndrome. Pediatricians were also more likely $(32 \%$ vs. $<2 \%$ of internists and family physicians) to correctly use a DNA-based test versus a karyotype to diagnose fragile $\mathrm{X}$ syndrome. When obstetrician/gynecologists were presented with a pregnant patient who had a family history of male mental retardation, $54 \%$ would obtain a two- or three-generation family history, $36 \%$ would discuss fragile $\mathrm{X}$ syndrome carrier testing, $64 \%$ would discuss fetal chromosome testing, and $69 \%$ would refer the patient for genetic counseling.

Pediatricians and obstetrician/gynecologists were more likely than internists and family physicians to know that reproductive risk is increased in a 25-yearold woman whose sister has a son with fragile $\mathrm{X}$ syndrome ( $49 \%$ vs. $21 \%, P<0.001$ ). Also, they were more likely to refer this woman for genetic counseling. When the scenario was changed to the patient's sister having a son with Down syndrome, across all specialties only $30 \%$ knew that the patient's reproductive risk for a child with Down syndrome was not increased over her age-specific risk. Pediatricians and obstetrician/gynecologists were not significantly more likely than internists and family physicians to refer this patient for genetic counseling to answer this question.

More than $95 \%$ of internists and obstetrician/gynecologists routinely asked about a family history of breast cancer, and more than $80 \%$ asked about ovarian cancer (family physicians were not queried about cancer inheritance). When a family history of breast or ovarian cancer was identified, $80 \%$ of internists and obstetrician/gynecologists completed a two- or three-generation pedigree, and more than $95 \%$ would "counsel the patient about risks based on family history" and "base recommendations for surveillance on the family history." Three percent of internists and $27 \%$ of obstetrician/gynecologists would refer the patient for a genetics consultation and $20 \%$ of both groups would refer the patient to another consultant. 
Internists and obstetrician/gynecologists were given the following scenario: "A 22-year-old woman had a grandmother (her mother's mother) who died of breast cancer and a 34-year-old aunt (her mother's sister) with bilateral breast cancer. She is seeking information about her odds of developing breast cancer." Fourteen percent of internists and obstetrician/gynecologists did not know that her risk is increased. Nevertheless, $75 \%$ of this group would provide risk counseling. Nine percent of internists and obstetrician/gynecologists would refer to a geneticist, $14 \%$ would refer to an oncologist or breast surgeon, and $22 \%$ would call a consultant or colleague.

This scenario was then modified, with the relatives affected with breast cancer being paternally, instead of maternally, related to the patient. Now, $57 \%$ of internists and obstetrician/gynecologists did not know that the patient's risk is increased, yet $55 \%$ of these practitioners reported that they would still provide risk counseling. Despite this deficiency in knowledge, physicians were no more likely to refer to a geneticist, oncologist, or breast surgeon or to call a consultant or colleague.

\section{DISCUSSION}

In this study, we explored the use and perceptions of genetics services by primary care physicians and their direct delivery of genetics services. We addressed differences between specialties. A few prior studies have examined these areas and none has looked at general perceptions of the benefits and detriments of genetics services and how perceptions might influence referral for these services.

\section{Utilization of genetics services}

In the Northwest, most primary care physicians reported having genetics services available to them, although internists were less knowledgeable about these services than were other specialists. Only $6 \%$ of providers reported additional needs for genetics services. Most consultations were obtained for a family history of a "genetic condition" or for individuals with dysmorphic features. Primary care physicians differed by specialty in how they use genetics services. Pediatricians and physicians who practiced obstetrics obtained genetics consultations at a much higher rate than did physicians who did not practice obstetrics.

Internists, although more likely to practice where genetics services are offered ( $53 \%$ practice in communities $>100,000$ ), are less likely to be aware of genetics services in their region and less likely to use these services than are other primary care physicians. Nearly one in six had no knowledge of services and reported no need for services. Fewer than half of internists routinely refer even for "traditional" genetic disorders such as polycystic kidney disease and Huntington's disease. Although this lower rate of use may be explained in part by the fact that many genetic disorders are evident in the prenatal and neonatal periods, we have demonstrated that internists are less likely than other specialists to perceive a benefit from genetics services and that the perception of limited or no benefit from genetics services is the leading deterrent to referral.

Providers with greater exposure to genetics problems are more knowledgeable about genetic disorders and tests. ${ }^{4}$ Physicians with more genetics knowledge were more likely to refer for consultation and were more likely to provide appropriate primary level genetics services to their patients. Consistent with observations of greater genetics knowledge among more recent medical school graduates, ${ }^{4}$ we found that this group was more likely to refer for genetics services than earlier graduates.

Managed care plan limits are a factor in deterring physician referrals for genetics services. This finding underscores the need for evidence of the value of genetics services and perhaps even their long-term cost-effectiveness. Given the increased penetration of managed care across the United States, this concern is likely to grow.

\section{Delivery of genetics services by primary care physicians}

Some genetics services are appropriately delivered in the primary care setting. ${ }^{11}$ There is no uniform agreement on just what comprises these services; however, we propose a definition of primary level genetics services in Table 4. Although many of these services are currently provided, our survey data indicate that knowledge and practice deficits still exist.

\section{Prenatal}

Maternal serum protein marker screening and fetal karyotyping were offered by more than $90 \%$ of

Table 4

Definition of primary level genetics services

- Identify individuals who may benefit from genetics services, including those with a genetic disorder and those at increased risk for having or transmitting a genetic disorder.

- Recognize historical and physical features of common genetic conditions and susceptibilities and features that suggest a genetic disorder.

- Monitor the health of individuals with a genetic disorder, in conjunction with genetics health professionals.

- Provide basic genetics information to patients and families in a culturally competent way using nondirective genetic counseling skills to facilitate comprehension and informed decision-making.

- Coordinate care for individuals with complex genetics service needs.

- Recognize the special psychosocial issues for a family in which one or more members is affected with a genetic disorder or susceptibility.

- Know how to access the full range of genetics services from which patients might benefit.

- Appropriately refer patients with additional genetics services needs.

- Facilitate use of genetics services. 
the prenatal care providers surveyed. In contrast, carrier screening for genetic disorders that are common in certain ethnic groups is offered routinely by only $70 \%$ of prenatal care providers, and preconceptional folic acid supplementation is offered routinely by only $78 \%$ of prenatal care providers. Yet both practices are recommended by the American College of Obstetrics and Gynecology ${ }^{12,13}$ and are considered standards of care. Although there is room for improvement, primary care providers have met one of the objectives of the family planning priority of Healthy People 2000: "Increase to at least 60 percent the proportion of primary care physicians who provide age-appropriate preconception care and counseling." 14

\section{Childhood}

To assess the delivery of primary level genetics services during childhood, we inquired about practices with respect to Down syndrome. It is appropriate to discuss a suspected diagnosis of Down syndrome with parents before there is karyotype confirmation, ${ }^{15}$ yet a significant fraction of providers would not do so. Furthermore, despite the necessity of a karyotype for accurate recurrence risk counseling for families of children with Down syndrome, many providers would not obtain one. Physicians often do not include an echocardiogram in their routine care of an infant with Down syndrome, despite the recommendation by the American Academy of Pediatrics. ${ }^{16}$ These findings indicate that more education is needed to inform primary care physicians about the optimal management of a newborn with Down syndrome.

\section{Adulthood}

In the scenarios presented, primary care physicians demonstrated a lack of understanding of inherited cancer risk. Even when uncertain or wrong about the relative risk, physicians chose to counsel the patient rather than refer. Although the repercussions of counseling based on inadequate knowledge may be difficult to measure, this practice is troubling nonetheless. Although it may be debatable who should provide risk counseling to patients and families with inherited susceptibility to a common disorder, it is clear that more primary care education and training in this area is needed. The Committee on Assessing Genetic Risks of the Institute of Medicine recommends that "genetics education and counseling tasks be analyzed to determine what level of complexity can appropriately be delivered by primary care providers and what degree of complexity requires the training and experience of specialized genetics personnel."17 In turn, it will become essential to evaluate the quality of services delivered by each of these groups.

\section{Attitudes of primary care physicians}

Referral to a medical geneticist relies on suspicion of a genetic or morphologic basis for a patient's disease, knowledge of available genetics services, and some familiarity with a medical geneticist's contribution to patient and family care. Also, physicians need to recognize the value of the service. Genetics professionals perceive their services to benefit patients even when no effective therapeutic intervention can be offered. Genetics evaluation often results in a specific diagnosis. Although this alone may help an individual or family, accurate prognostic and recurrence risk information can be provided only when a diagnosis is established. Given our finding that the most important factor prompting a genetics referral was the patient's or family's interest in the referral, it seems that consumers do, in fact, often drive referrals for genetics services. A perceived detriment of genetics services was "creating undue worry", hence commitment of physicians not to do harm may deter some referrals. However, this is a detriment that is perceived by physicians and, in fact, may not reflect patients' perceptions. Benefits of genetics services may be more evident to consumers than to physicians. ${ }^{18,19}$ Primary care physicians need increased awareness of the genetic information needs of their patients.

This study considered the attitudes of physicians toward genetics services in the context of a referral to a genetics consultant. In fact, many genetics professionals currently work in multidisciplinary settings, such as cancer clinics, sickle cell disease clinics, cystic fibrosis clinics, and obstetrical practices. This may be a successful paradigm for improved delivery of genetics services in the future. Also, physicians may perceive greater value in genetics services that are integrated into comprehensive care.

\section{Study limitations}

Despite the limited response rate to this survey, we have established that the demographics of our more than 1600 respondents are representative of the target study population. Physicians with a greater interest in genetics, more current knowledge, and more experience in delivering and referring to genetics services may have been more likely to respond. If this group of physicians is indeed over-represented, then one would expect our results to be skewed toward higher rates of knowledge and utilization. Regardless, our study lays important groundwork for further investigations.

\section{CONCLUSIONS}

Primary care physicians need to move beyond their traditional view of the geneticist as someone who treats only patients with rare and esoteric disorders. The findings of this survey indicate that primary care physicians do not use geneticists or genetic counselors as a resource in the care of many individuals with genetics-influenced or frank genetic disorders. Indeed, Hall et al. ${ }^{20}$ have suggested that a significant number of people who might benefit from genetics services are not referred. As genetic factors in common diseases are identified and a whole new array of gene-based therapies are developed, the modern geneticist will play an integral role in the care of an increasing number of patients.

Although the traditional medical model focuses on treatment of disease, the principal value of most genetics services is to educate patients and providers, facilitate informed reproductive decision making, and prevent complications of disease. These services have long been heralded as beneficial by consumers of genetics services ${ }^{18,19}$; however, there have been few studies of whether providers value these services. ${ }^{10}$ In this study, we have shown that a substantial percentage of primary care physicians do not value genetics consultation. This lack of recognition of genetics as important or beneficial has shocking and far-reaching implications and represents the 
greatest challenge to the development of effective modes of remediation. However, it cannot be assumed that the providers who do not see a benefit are always wrong. As medical genetics moves into a more mainstream position in health care, the impact of genetics services on disease outcomes and health care costs will need to be evaluated. Genetics professionals must meet the challenge of trying to document the value of their services.

Since we completed data collection substantial resources have been directed to primary care physician education in genetics. ${ }^{21}$ Unfortunately, courses for primary care physicians that are devoted entirely to genetics have had limited impact and often have been poorly attended. Instead, genetics must be recognized and taught as an integrative discipline, with inclusion of genetics in every continuing medical education course. New educational programs must go beyond imparting knowledge; real success will be measured by changes in perceptions and utilization of genetics services.

\section{Acknowledgments}

This study was funded by the Pacific Northwest Regional Genetics Group (PacNoRGG) (MCJ141100213) and the Regional Program of Genetics Education for Primary Care Providers (MCJ41 1005) (S.J.H. and M.P.E.), Maternal and Child Health Bureau, Department of Health and Human Services.

\section{References}

1. Online Mendelian Inheritance in Man, OMIM. Center for Medical Genetics, Johns Hopkins University (Baltimore, MD) and National Center for Biotechnology Information, National Library of Medicine (Bethesda, MD), 1996.

2. Baird PA, Anderson TW, Newcombe HB, Lowry RB. Genetic disorders in children and young adults: A population study. Am J Hum Genet 1988;42:677-693.

3. Firth HV, Lindenbaum RH. UK clinicians' knowledge of and attitudes to the prenatal diagnosis of single gene disorders. JMed Genet 1992;29:20-23.

4. Hofman KJ, Tambor ES, Chase GA, Geller G, Faden RR, Holtzman NA. Physicians' knowledge of genetics and genetic tests. Acad Med 1993;68:625-632.

5. Stratakis CA, Cavuto NJ, Nelson D, Rennert OM. Molecular genetics in pediatric training: How much do we really know? Md Med J 1995;44:210-213.
6. Giardiello FM, Brensinger JD, Petersen GM, Luce MC, Hylind $\mathrm{LM}, \mathrm{Bacon} \mathrm{JA}$ et al. The use and interpretation of commercial APC gene testing for familial adenomatous polyposis. N Engl J Med 1997;336:823-827.

7. Naylor EW. Genetic screening and genetic counseling: Knowledge, attitudes, and practices in two groups of family planning professionals. Soc Biol 1975;22:304-314.

8. Weitz R. Barriers to acceptance of genetic counseling among primary care physicians. Soc Biol 1979;26:189-197.

9. Young ID. Undergraduate teaching of genetics. Med Educ 1984;18:151-154.

10. Guilbert P, Cheater F. Health visitors' awareness and perception of clinical genetic services. I Med Genet 1990;27:508-511.

11. Hayflick SJ, Eiff MP. The role of primary care providers in the delivery of genetics services. Community Genet 1998;1:18-22.

12. Anonymous. ACOG educational bulletin. Maternal serum screening. Number 228, September 1996 (replaces no. 154, April 1991). Committee on Educational Bulletins of the American College of Obstetricians and Gynecologists. Int J Gynaecol Obstet 1996;55:299-308.

13. Anonymous. ACOG educational bulletin. Nutrition and women. Number 229, October 1996. Committee on Educational Bulletins of the American College of Obstetricians and Gynecologists. Int J Gynaecol Obstet 1997;56:71-81.

14. Anonymous. Healthy People 2000: National health promotion and disease prevention objectives (excerpts). US Public Health Service. I Allied Health 1990, revised 1997;19:297-311.

15. Cooley WC, Graham JM Jr. Down syndrome-an update and review for the primary pediatrician. Clin Pediatr 1991;30:233-253.

16. Anonymous. Health supervision for children with Down syndrome. American Academy of Pediatrics Committee on Genetics. Pediatrics 1994;93:855-859.

17. Andrews LB, Fullarton JE, Holtzman NA, Motulsky AG. Assessing genetic risks: Implications for health and social policy. Washington, DC: National Academy Press, 1994;338.

18. Black R, Weiss J. Gaps in service: Patient/family perspective. Md Med J 1989;38:933-937.

19. Middleton L, Smith A. Consumer interests in quality genetics services. Presented at the Fourth Joint Clinical Genetics Meeting, 28th Annual March of Dimes Clinical Genetics Conference. Fort Lauderdale, Florida, 1997.

20. Hall J, Powers E, McIlnaine R, Ean V. The frequency and familial burden of genetic disease in a pediatric hospital. Am J Med Genet 1978;1:416-436.

21. Council of Regional Networks in Genetics (CORN). Maternal and Child Health Bureau, Conference on Genetics Education in Primary Care. Washington, DC, 1996. 


\section{APPENDIX 1}

\section{Internal Medicine Genetics Survey}

For the purpose of this survey, genetics refers to heritable diseases, birth defects, and inborn errors of metabolism. Please answer the following questions about yourself and your practice.

For Question 1, circle one answer for each item.

1) a. In your practice, do you routinely take a family history?

$\begin{array}{cc}\text { YES } & \text { NO } \\ 1 & 2 \\ \text { YES } & \text { NO } \\ 1 & 2 \\ 1 & 2 \\ \text { YES } & \text { NO } \\ 1 & 2 \\ 1 & 2 \\ 1 & 2 \\ 1 & 2\end{array}$

2) In your practice, how often do you ask your patients if they have a family history of: (circle one number for each)

If YES, when do you usually tirst take a family history?

b. routine basis early in care of the patient

c. as needed basis to assess a specific problem

If YES, what does the history usually include?

d. completion of a standard form or checklist

e. a two or three generation family history

f. the family's ethnic background

g. medical risk factors (c.g., hypercholesterolemia, cancer)

b. ovarian cancer 112

3) For a patient with a family history of breast and/or ovarian cancer, how often do you:

$$
\text { (circle one number for each item) }
$$

a. complcte a two or three gencration family history

b. counsel the patient about risks based on family history

c. basc your recommendations for surveillance on the family history

USUALLY SOMETIMES RARELY

d. refer them for genetics consultation

e. refer them to other consultant (specify)

$\begin{array}{ccc}1 & 2 & 3 \\ 1 & 2 & 3 \\ 1 & 2 & 3 \\ 1 & 2 & 3 \\ 1 & 2 & 3\end{array}$

4) In your practice, how do you evaluate a male with isolated mental retardation:
(circle one number for each item)
USUALLY SOMETIMES RARELY
a. complete a two or three generation family history
b. refer the patient to a genetics clinic
c. obtain chromosome testing
d. obtain fragile $X$ syndrome testing
e. other (specify)

$\begin{array}{lll}1 & 2 & 3 \\ 1 & 2 & 3 \\ 1 & 2 & 3 \\ 1 & 2 & 3 \\ 1 & 2 & 3\end{array}$
f. If you test for fragile $X$ syndrome, what type of testing do you use? KARYOTYPE 1 IDNA-BASED 2

5) Of patients with the following, would you refer for a genetics consultation:

$\begin{array}{lccc}\text { (circle one for each) } & \text { USUALLY } & \text { SOMETIMES } & \text { RARELY } \\ \text { a. cystic kidney disease } & 1 & 2 & 3 \\ \text { b. isolated mental retardation } & 1 & 2 & 3 \\ \text { c. family history of cancer } & 1 & 2 & 3 \\ \text { d. birth defects } & 1 & 2 & 3 \\ \text { e. deafness } & 1 & 2 & 3 \\ \text { f. congenital heart defect } & 1 & 2 & 3 \\ \text { g. family history of Huntington disease } & 1 & 2 & 3\end{array}$

6) a. Is genetics counseling or consultation available to you'? (circle one) YES 1 NO 2 DONT KNOW 3

b. If YES, by whom? (circle all that apply) YES NO)
(1) Medical geneticist
(2) Obstetrician or perinatologist
(3) Genetics counselor
(4) Other (specify)
2
2 


\section{APPENDIX 1 continued}

6) c. How far away from you is this consultant? (circle the number next to the closest estimate)
(1) within 30 minute drive
(3) 1 - 3 hour drive
(2) 30 - 60 minute drive
(4) $>3$ hour drive

$\begin{array}{lllll}\text { 7) a. Do you have needs for genetics services beyond those presently available to you (circle } & \text { one) } & \text { YES } & 1 & \text { NO } 2\end{array}$ b. If Yes, what is needed?

8) Approximately, how many genetics consultations have you obtained in the past 12 months?
a. none
b. $1-5$
c. 6-10
d. 11-15
e. 16 or more

9) When you request a genetics consultation, how important are the following factors in your decision to refer? (circle one number for each item)
a. patient's interest in the assessment
b. severity of the disorder
c. availability of treatment
d. desire for management recommendations
e. family's desire for recurrence information
f. malpractice concerns

$\begin{array}{ccc}\text { VERY } & \text { SOMEWHAT } & \text { NOT AT ALL } \\ 1 & 2 & 3 \\ 1 & 2 & 3 \\ 1 & 2 & 3 \\ 1 & 2 & 3 \\ 1 & 2 & 3 \\ 1 & 2 & 3\end{array}$

10) When you choose not to obtain a genetics consultation, how often is each of the following the reason? (circle one number for each item) USUALLY SOMETIMES RARELY NEVER
a. distance to consultant too great
b. don't see much benefit for the patient
c. able to get info from a consultant by phone
d. managed care plan limits
e. other (specify)

$\begin{array}{ll}1 & 2 \\ 1 & 2 \\ 1 & 2 \\ 1 & 2 \\ 1 & 2\end{array}$

$\begin{array}{lll}2 & 3 & 4 \\ 2 & 3 & 4 \\ 2 & 3 & 4 \\ 2 & 3 & 4 \\ 2 & 3 & 4\end{array}$

11) What do you perceive to be the benefits of a genetics consultation? (if none, write "none")

12) What do you perceive to be the detriments of a genetics consultation" (if none, write "none")

13) Which of the following educational resources do you use to update your medical knowledge?
a. hospital conferences
b. review courses
$1 \quad 2$
c. satellite teleconferences

12
d. other (specify)

14) Would you use a free on-line computer resource to further your genetics knowledge or find information you need about genetic conditions? (circle one)
a. Definitely
b. Probably
c. Probably not
d. Definitely not

15) A 22 year old woman had a grandmother (her mother's mother) who died of breast cancer and a 34 yr old aunt (her mother's sister) with bilateral breast cancer. She is seeking information about her odds of developing breast cancer. (circle one)
u. Is her risk of breast cancer increased over the general population?
b. Would you counsel her about her risk?
c. Would you refer for genetics consultation to answer this question?
d. Would you refer to an oncologist or breast surgeon to answer this question? $1 \quad 2 \quad 3$
e. Would you call a consultant or colleague to answer this question?

$\begin{array}{clc}\text { YES } & \text { NO } & \text { UNSUR } \\ 1 & 2 & 3 \\ 1 & 2 & 3 \\ 1 & 2 & 3 \\ 1 & 2 & 3 \\ 1 & 2 & 3\end{array}$

YES NO UNSURE 
16) A 22 year old woman had a grandmother (her father's mother) who died of breast cancer and a 34 yr old aunt (her father's sister) with bilateral breast cancer. She is seeking information about her odds of developing breast cancer. (circle

a. Is her risk of breast cancer increased over the general population?

b. Would you counsel her about her risk?

c. Would you refer for genetics consultation to answer this question?

d. Would you refer to an oncologist or breast surgeon to answer this question?

e. Would you call a consultant or colleague to answer this question?

$\begin{array}{ccc}\text { YES } & \text { NO } & \text { INSUR } \\ 1 & 2 & 3 \\ 1 & 2 & 3 \\ 1 & 2 & 3 \\ 1 & 2 & 3 \\ 1 & 2 & 3\end{array}$

17) A 25-year-old woman has a nephew (her sister's son) with fragile $X$ syndrome. $\underset{\text { YES }}{\text { circle }} \begin{aligned} & \text { one) } \\ & \text { NO }\end{aligned}$ UNSURF:

a. Is her risk of having a child with fragile $X$ syndrome increased? $\quad 1 \quad 2 \quad 3$

b. Would you refer this woman for a genetics consultation to answer this question? $1 \quad 1 \quad 2 \quad 3$

c. Would you call a consultant or colleague to answer this question? $\quad 1 \quad 2 \quad 3$

18) A 25-year-old woman has a nephew (her sister's son) with Down syndrome due to trisomy 21 . (circle one)

a. Is her risk of having a child with Down syndrome increased?

YES NO NOT SURE

b. Would you refer this woman for genetics consultation to answer this question"

c. Would you call a consultant or colleague to answer this question?

one)

Y) Year you graduated from medical school: 19

20) State in which you practice

21) Are you internal medicine residency trained? (circle one) YES I

NO 2

22) Your Gender (circle) Female Male

23) Size of your community (circle one)
(1) $0-10.000$
(2) $10,001-50,000$
(3) $50,001-100,000$
(4) $100,001-500,000$
(5) $>500,000$

24) Practice Size: (if you practice in a group, estimate the number of patients in your own panel) (circle one)
(1) $0-500$
(3) $1001-2500$
(2) $501-1000$
(4) $2501-5000$
(5) $>5000$

25) Did you have a genetics course (NOT embryology) in medical school? YES I

NO 2 DONT KNOW 3

26) Have you attended any CME courses specifically dealing with genetics? YES

NO 2

27) Wc welcome your additional thoughts and comments.

Thank You Very Much For Your Time! 